followed did not progress to AIDS until 108 months. Independent prognostic factors for AIDS-free-time were: treatment with ART without HAART (HR 2.1; 95\% CI 1.6 to 2.8), no treatment regimen (HR 3.0; 95\% CI 2.5 to 3.6); age at HIV infection diagnosis between 30 and 49 years (HR 1.2; 95\% CI 1.1 to 1.3 ), age over 50 years (HR 2.9; $95 \%$ CI 2.3 to 5.2 ); black race/colour (HR 1.4; $95 \%$ CI 1.1 to 1.7 ); MSM (HR 1.4; 95\% CI 1.1 to 1.6) and IDU (HR 1.7; 95\% CI 1.3 to 2.2) exposure categories; up to 8 years of schooling (HR 1.3; $95 \% \mathrm{CI}$ 1.1 to 1.5 ) and no schooling (HR 2.0; 95\% CI 1.4 to 5.6); and CD4 count between 350 and 500 cells $/ \mathrm{mm}^{3}$ (HR 1.6; 95\% CI 1.3 to 1.9). Conclusions Increased AIDS-free-time was observed, with HAART. Decrease in the incidence rates were observed, Predictor factors to AIDS were treatment, age, race/colour, transmission categories, schooling and CD4 count.

\section{SP3-46 AIDS SURVIVAL IN THE PRE AND POST-HAART ERAS IN THE SAO PAULO AIDS COHORT, BRAZIL}

doi:10.1136/jech.2011.1429760.46

${ }^{1} \mathrm{M}$ V Tancredi, ${ }^{2} E$ A Waldman. ${ }^{1}$ Sao Paulo State STD and AIDS Training and Reference Center, Sao Paulo, Sao Paulo, Brazil; ' 2 School of Public Health of Sao Paulo University, Sao Paulo, Sao Paulo, Brazil

Background AIDS remains a great public health problem and the effect of ART has been studied. The objectives were to estimate AIDS mortality rates, median survival time and to investigate death predictor factors.

Methods Retrospective cohort study, with 6594 adult patients followed from 1988 to 2005. The Kaplan-Meier estimator, the Cox proportional hazard model and HRs estimates were used.

Results In a follow-up of 203008 persons-year, 2936 patients progressed to death. AIDS mortality rates were 17.6, 23.2, and 7.8 person-years in the 1988-1993, 1994-1996 and 1997-2003 periods, respectively. Median progression time from AIDS to death was 13.4 months in the 1988-1993 period; 22.3 months, between 1994 and 1996, and in the 1997-2003 period, over 50\% of patients followed survived. Independent predictor factors for death were: AIDS diagnosis period 1994-1996 (HR 2.0; 95\% CI 1.8 to 2.2) and 1988-1993 (HR 3.2; 95\% CI 2.8 to 3.5); AIDS diagnosis age between 30 and 49 years (HR 1.4; 95\% CI 1.2 to 1.5), age over 50 (HR 2.0; $95 \%$ CI 1.7 to 2.3 ); MSM (HR 1.1; 95\% CI 1.1 to 1.2 ) and IDU (HR 1.5 ; $95 \%$ CI 1.3 to 1.6$)$ exposure categories; up to 8 years of schooling (HR 1.4; 95\% CI 1.3 to 1.5) and no schooling (HR 2.1; 95\% CI 1.6 to 2.8); and CD4 count between 350 and 500 cells $/ \mathrm{mm}^{3}$ (HR $1.2 ; 95 \%$ CI 1.1 to 1.2 ) and $<350$ cells $/ \mathrm{mm}^{3}$ (HR 1.3; 95\% CI 1.2 to 1.3 ). Conclusions Increase in AIDS survival and decrease in the mortality rates were observed with HAART. Predictor factors to death were AIDS diagnosis period, age, transmission categories, schooling and CD4 count. The results show the great positive impact of the Brazilian National AIDS Program.

\section{SP3-47 SPATIAL PROXIMITY AND CHILDHOOD HOSPITAL ADMISSIONS IN A DENSELY POPULATED CONURBATION: EVIDENCE FROM HONG KONG'S “CHILDREN OF 1997" BIRTH COHORT}

doi:10.1136/jech.2011.1429760.47

C M Schooling, C Yau, M K Kwok, ${ }^{*}$ B J Cowling, T H Lam, G M Leung. Life course and Lifestyle Epidemiology Group, School of Public Health, Li Ka Shing Faculty of Medicine, The University of Hong Kong, Hong Kong SAR, China

Introduction Physical distance is a barrier to hospital utilisation. In a very densely populated city in China, we examined whether use of public hospitals by children was associated with individual-level residential proximity, and whether these associations varied with type of admission.
Methods The authors used multivariable negative binomial regression in a large, population-representative birth cohort to examine the adjusted associations of proximity to hospitals with Accidents and Emergency services, proxied by distance to the nearest such hospital, with hospital admissions, bed-days and average length of stay from 8 days to 8 years of age.

Results Physical proximity was positively associated with emergency admissions in children (incidence rate ratio (IRR) $1.23,95 \%$ CI 1.11 to 1.35 for $<1 \mathrm{~km}$ compared to $\geq 2 \mathrm{~km}$ ) and bed-days but not with average length of stay, adjusted for age, sex and socio-economic position. However, in a similar comparison there was no such association for other (ie, planned) admissions (IRR 1.04, 95\% CI 0.85 to 1.27).

Conclusion Proximity was associated with hospital use for emergency admissions. Given the societal costs of such use and the risks of iatrogenesis, attention should focus on achieving a more effective use of scarce resources.

\section{SP3-48 ROUTINE MORTALITY AND CAUSE OF DEATH REPORTING AND ANALYSIS SYSTEMS IN SEVEN PACIFIC ISLAND COUNTRIES}

doi:10.1136/jech.2011.1429760.48

${ }^{1} \mathrm{~K}$ Carter, ${ }^{* 1} \mathrm{C}$ Rao, ${ }^{1} \mathrm{~A}$ Lopez, ${ }^{1,2} \mathrm{R}$ Taylor. ${ }^{1}$ School of Population Health, University of Queensland, Herston (Brisbane), Queensland, Australia; ${ }^{2}$ School of Public Health and Community Medicine, University of New South Wales, Sydney, New South Wales, Australia

Introduction Statistics on mortality levels and causes of death are essential for health planning. However, at the end of 2003, only 7 of 27 countries in the Western Pacific Region had data available on causes of death. Routine death reporting systems across seven Pacific Island Countries; Fiji, Kiribati, Nauru, Palau, Solomon Islands, Tonga and Vanuatu, are examined. Strengths and limitations common across national systems are identified, and system characteristics related to data availability and quality.

Methods System assessments included key informant interviews, observation of processes, and document review. Findings were grouped according to a framework that classifies system characteristics according to societal issues, the national administrative environment, administration, technical and ownership issues.

Results Routine reporting of deaths is predominantly managed through civil registration systems or within Health departments. Health reporting systems are critical in supporting the civil registration process. Significantly more information is available than currently used. Legislation on death reporting exists for all islands, but does not necessarily reflect current practices. Significant duplication of data collection and entry exists across all systems. The close interaction between health staff and local communities could provide a good foundation for further improvement in death reporting in these countries. Responsibility, authority and ownership were central to the sustainability of the reporting systems.

Conclusion For Pacific Island Countries to effectively address health challenges there is no substitute for routine mortality and cause of death data collections. Suitable systems exist, but need to be strengthened to improve the completeness and quality of the data available.

SP3-49 THE RELATIONSHIP OF CLASS CLOSURE LENGTH AND THE CHANGE OF ABSENTEES AT ELEMENTARY SCHOOLS IN THE 2009 A/H1N1 INFLUENZA EXPANSION IN JAPAN: THE ANALYSIS IN T CITY, IBARAKI PREFECTURE

doi:10.1136/jech.2011.1429760.49

${ }^{1} \mathrm{~S}$ Yamamoto, ${ }^{*} \mathrm{H}$ Takahashi, ${ }^{3}$ I Wada, ${ }^{4} \mathrm{~A}$ Uda, ${ }^{5} \mathrm{~F}$ Irie. ${ }^{1}$ School of Medicine, School of Medicine and Medical Sciences, University of Tsukuba, Tsukuba, Ibaraki, 
Japan; ${ }^{2}$ Department of Epidemiology, University of Tsukuba, Tsukuba, Ibaraki, Japan; ${ }^{3}$ Social Services Division, Southern District Head Branch, Ibaraki Prefectural Government, Tsuchiura, Ibaraki, Japan; ${ }^{4}$ Graduate School of Comprehensive Human Sciences, Master's Program in Medical Sciences, University of Tsukuba, Tsukuba, Ibaraki, Japan; ${ }^{5}$ Health Services Disease Control Division, Department of Health and Social Services, Ibaraki Prefectural Government, Mito, Ibaraki, Japan

Introduction The $2009 \mathrm{~A} / \mathrm{H} 1 \mathrm{~N} 1$ influenza spread also in Japan. Many students were absent in elementary schools, To prevent its expansion, many school decided class closure with their original length under rough guideline by local education board. However, its effect had not been clear. The objective was to examine whether or not the class closure length related to the change of absentees.

Methods Subject was all the classes in elementary schools in T city that class closure was carried in the period from 1 September to 24 December in 2009. We sent the questionnaire including the questions (1) the number of students in class, (2) the number of absentees on the day, (3) whether or not class closure was carried out on the day, to the school principal, and asked school nurses to write under each class attendance book and to return them by post mail. The length of class closure and the change of absentees before and after class closure was analysed by $\chi^{2}$ test with statistical soft R2.11.1

Results 16 of total 37 elementary schools replied (43.2\%), and 103 classes of 15 schools with the closure were analysed. It revealed statistical associations between class closure length and the change of the proportions of absentee $(\mathrm{p}<0.001)$, and between class closure length and the proportions of class that absentee was decreased $(\mathrm{p}<0.001)$

Conclusion There were tendencies that the number of absentee decreased after class closure, and the longer class closure days, the fewer absentees changed.

\section{SP3-50 SERUM HEPATOCYTE GROWTH FACTOR LEVELS AND MORTALITIES FROM CANCER IN APPARENTLY HEALTHY GENERAL POPULATION}

doi:10.1136/jech.2011.1429760.50

\section{SP3-51 ACCESS TO TB PATIENTS OF DIRECTLY OBSERVED THERAPY (DOTS) DURING NATURAL DISASTERS IN BIHAR, INDIA}

doi:10.1136/jech.2011.1429760.51

R Kumar.* Integrated Disease Surveillance Project, Pauri, Uttarakhand, India

Introduction This pilot study attempts to look at pattern and problem of adherence to DOTS by TB patient during flooding in Bihar, India.

Methods A cross-sectional survey was undertaken among 21 (female $\mathrm{n}=9$ ) TB patients who were participating in DOTS and faced the problem of flooding in Muzaffarpur district of Bihar, India. They were interviewed to collect information on age, sex, education, occupation, duration of flooding in the village, discontinuity period due to flooding and other factors, change in DOT provider, and knowledge on importance of continuing treatment using a semistructured questionnaire.

Results Mean duration of flooding was 92.9 days (SD 32.4). Mean discontinuity period due to flooding was 26.9 days (SD 24.0). In the Fisher's Exact Test, Female TB treatment beneficiaries were found to more affected than males $(\mathrm{p}<0.005)$. Following discontinuing DOTS, $3(14.3 \%)$ participants thought that their disease would not be cured, 8 (38.1\%) participants thought their TB disease would come back, whereas and $9.5 \%$ said they did not know.

Conclusion Although flooding lead to discontinuity in treatment to many of the TB patients on DOTS. This may lead to antituberculosis drug resistance. The finding that females discontinued treatment more frequently than males needs to be highlighted. The TB control programme should look further in to this and take appropriate measures to address the issue.

\section{SP3-52 A DIFFERENCE IN ADRS (ADVERSE DRUG REACTIONS) MORTALITY RATE IN THAI TUBERCULOSIS PATIENTS BETWEEN YEAR 2008 AND 2009}

doi:10.1136/jech.2011.1429760.52

S Wechwithan, ${ }^{*}$ P Sriphiromya. Health product Vigilance Center, Nonthaburi, Thailand

Background Surveillance of adverse drug reactions in Thailand is conducted through the spontaneous voluntary reporting system by hospital pharmacists and healthcare professionals. Adverse drug reactions reports have been collected in national spontaneous reporting database called Thaivigibase since year 1985. Public health program using medicine in AIDS, Tuberculosis (TB) control program have collected the patients' records. Integrating public health program in TB patients and spontaneous reporting system can receive $\mathrm{ADR}$ mortality rate compare difference in anti-tuberculosis drug group. This $\mathrm{ADR}$ mortality rate may reflect $\mathrm{TB}$ drug group safety surveillance system.

Objective This study is aimed to compare difference in ADRs mortality rate in Thai tuberculosis patients, between year 2008 and 2009.

Study Design Descriptive observational study design is used for this study.

Materials and Methods Adverse reaction reports of patients to anti tuberculosis drugs from Thaivigibase and $\mathrm{TB}$ patient disease surveillance database from Bureau of Epidemiology during year 2008-2009 were retrieved and calculated. The pattern of spontaneous fatal adverse reactions to anti-tuberculosis drugs were described by analysing the data from Thaivigibase between year 2008 and 2009.

Results/Conclusion ADRs mortality rates to anti-tuberculosis drugs were 1.97 per 1000 patients in year 2009 compared with 4.35 per 1000 patients in year 2008. Stevens-Johnson Syndrome and 